\title{
FLARE PUFFS AS A CAUSE OF TYPE III RADIO BURSTS
}

\author{
R. G. GIOVANELLI
}

Division of Physics, National Standards Laboratory, Sydney, Australia

It has been well established that a strong association exists between flares and type III bursts: Loughhead, Roberts, and McCabe [1] showed that 60 to 70 per cent of type III bursts occurred during the lifetimes of flares or microflares of apparent area exceeding about $20 \times 10^{-6}$ solar hemispheres. On the other hand, only about 22 per cent of all such flares are associated with bursts. This low figure indicates that there are differences of degree or type between various flares, but the large area range involved shows that flare size alone is not the determining factor.

About one-quarter of all flares emit surges that can be observed on the disk because of their darkness in $\mathrm{H} \alpha$. Loughhead, Roberts, and McCabe pointed out that in the period covered by their analysis, type III bursts occurred with 29 per cent of flare-surges, and with only 18 per cent of flares not accompanied by observed surges; but that these differences lacked statistical significance.

The first trace of a surge, however, is usually the ejection of diffuse matter, brighter than the chromosphere, which fades and disappears by becoming transparent, the dark surge subsequently developing and traveling outward in the same direction [2]. Some of these ejections appear to be of an explosive character, the flare expanding rapidly within a minute or so. Usually, but not always, this expansion or "puff" occurs at the outbreak of the flare. Its sudden and violent nature suggests that a puff may be the observable flare characteristic most likely to be associated with type III bursts.

To study this, I have selected 27 flares with well-defined puffs occurring during times of radio spectrograph observation. Of these, 18 (or 67 per cent) coincided with type III bursts to within \pm 2 minutes. The result is of high statistical significance, and establishes that flares with puffs are much more closely associated with type III bursts than are other flares.

It is interesting to note that in the above flares about one-half of the areas were below $40 \times 10^{-0}$ hemispheres, and again there appeared to be no significant dependence of type III burst associations on size. With one exception, all the flares produced dark surges. Thus it would appear that the one explosion, the flare-puff, ejects the surge-particle stream at a velocity of the order of $100 \mathrm{~km} / \mathrm{second}$ besides the much faster disturbance causing the type III burst.

\section{REFERENCES}

[1] Loughhead, R. E., Roberts, J. A., and McCabe, Marie K. Aust. J. Phys. 10, 483, 1957.

[2] Giovanelli, R. G., and McCabe, Marie K. Aust. J. Phys. 11, 191, 1958. 